\title{
Effect of Surface Mechanical Attrition Treatment on the very high cycle fatigue behavior of TC11
}

\author{
Tao Gao ${ }^{1}$, Zhidan $\mathrm{Sun}^{2}$, Hongqian Xue ${ }^{{ }^{*}}$, and Delphine Retraint ${ }^{2}$ \\ ${ }^{1}$ School of mechanical engineering, Northwestern Polytechnical University, Xi' an 710072 China \\ ${ }^{2}$ ICD, P2MN, LASMIS, University of Technology of Troyes, CNRS, Troyes, France
}

\begin{abstract}
As an important engine component material, TC11 (Ti-6.5Al-3.5Mo-1.5Zr-0.3Si) titanium alloy is subjected to high frequency cyclic loading and its failure occurs beyond $10^{9}$ cycles. It is thus essential to investigate the very high cycle fatigue (VHCF) behavior of this alloy. Surface Mechanical Attrition Treatment (SMAT) is a promising surface treatment technique to improve fatigue strength by modifying the surface microstructure. Therefore, it is important to understand the fatigue damage and failure process of SMATed titanium alloy in the VHCF regime. In this work, VHCF tests of TC11 before and after SMAT under fully reversed loading were conducted at room temperature by using an ultrasonic fatigue testing machine at a frequency of $20 \mathrm{kHz}$. The preliminary results seem to indicate that SMAT can reduce fatigue strength and fatigue life of TC11. Fracture surface analysis of the specimens before and after SMAT was performed using scanning electron microscope (SEM) to investigate the mechanisms of crack initiation and propagation. Particular attention was paid to fatigue crack initiation sites. The effect of SMAT on damage mechanism of SMATed TC11 in the VHCF regime was discussed.
\end{abstract}

\section{Introduction}

Many titanium components used in the field of aeronautical engineering such as blade, compressor and gear, are subjected to high frequency cyclic loading and failure occurs beyond $10^{7}$ cycles and even up to $10^{11}$ cycles. This fatigue cycle range is called very high cycle fatigue (VHCF) regime. Beyond the fatigue limit determined using conventional fatigue testing method, many investigations of titanium alloy in the VHCF regime show S-N curves with stepped shape, which continuously decreases with significant slope at cyclic loading cycles greater than $10^{7}$ cycles [1-3]. Moreover, the crack initiation site is transferred from the surface to the subsurface or even the interior of materials in the VHCF regime [1, 4], which implies that the damage and failure mechanism in VHCF could be different from those in low cycle fatigue (LCF) and high cycle fatigue (HCF). Therefore, understanding the fatigue damage and failure process of titanium alloy in the VHCF regime is important for ensuring reliability and safety of aircrafts during the period of service.

It is well known that engineering components are subjected to fatigue loading and failure from flaws or cracks at the surface. Surface modification techniques have been successfully applied to improve the fatigue strength of materials by forming a hardened surface layer and introducing superficial compressive residual stresses. Surface Mechanical Attrition Treatment (SMAT) is a relatively new method of surface severe plastic deformation (SPD), which is attracting increasing attention [5]. SMAT process is similar to conventional shot peening except that the shot present a larger diameter in the case of SMAT and are propelled by a sonotrode vibrating at ultrasonic frequency instead of compressed air. The main beneficial characteristic of the SMAT process is to transform ordinary coarse grains to nano-sized grain in the surface layer of a part without changing the chemical composition of materials [6]. The SMAT process has been proved to be efficient to nanocrystallize surface layer of various materials [7] including aluminum, stainless steels and titanium alloys [8-11]. Extensive research has shown that fine grains can improve the plastic strength and inhibit crack initiation, while coarse grains exhibit better crack propagation resistance due to their good ductility and toughness [12]. Considering the fact that most fatigue cracks of components initiate at the surface and propagate inward,

Corresponding author: xuedang@nwpu.edu.cn 
a nanostructured surface layer and coarse grained interior bulk can be expected to effectively improve fatigue life. Furthermore, the compressive residual stresses and work hardening induced by SMAT can also considerably delay fatigue crack initiation and propagation.

It has been demonstrated that SMAT can improve the fatigue life of different materials [13, 14]. However, most studies focused on low and high cycle fatigue improvement and considered the effect of surface residual stresses, roughness and microstructures induced by surface treatment. Only few investigations focus on the application of surface treatment in the VHCF regime and no study on the VHCF properties of SMATed titanium alloy has been reported in the literature.

This paper is dedicated to investigate the VHCF performance of a TC11 titanium alloy before and after SMAT. Ultrasonic fatigue tests at a frequency of $20 \mathrm{kHz}$ are conducted to examine the VHCF properties of TC11. Two series of dumbbell-shaped ultrasonic fatigue specimens, mechanically polished and SMATed respectively, are prepared to investigate the fatigue behavior and to study the effect of SMAT on fatigue life under VHCF. Fracture surfaces are analyzed by scanning electron microscope (SEM) in order to examine the mechanism of crack initiation and propagation involved during fatigue loading. The influence of SMAT on the fatigue behavior of the untreated and SMATed specimens is then discussed.

\section{Material and experimental methods}

The material used in this work is an $\alpha+\beta$ titanium alloy, TC11 (Chinese standard) in a mill-annealed condition. The chemical composition of this alloy is shown in Table 1. This alloy has a duplex microstructure, which contains about $60 \%$ volume fraction of primary $\alpha$ phase. The typical values of basic physical/mechanical properties are shown in Table 2.

Table 1. Chemical composition of TC11 (in weight-\%)

\begin{tabular}{cccccc}
\hline $\mathrm{Al}$ & $\mathrm{Mo}$ & $\mathrm{Zr}$ & $\mathrm{Si}$ & $\mathrm{Fe}$ & $\mathrm{Ti}$ \\
\hline 6.45 & 3.45 & 1.69 & 0.24 & 0.01 & Bal. \\
\hline
\end{tabular}

Table 2. Basic physical/mechanical properties of TC11

\begin{tabular}{cccc}
\hline$\rho\left(\mathrm{kg} \cdot \mathrm{m}^{-3}\right)$ & $\begin{array}{c}\mathrm{UTS} \\
(\mathrm{MPa})\end{array}$ & $R_{\mathrm{p} 0.2}(\mathrm{MPa})$ & $\mathrm{A} \mathrm{( \% )}$ \\
\hline 6.45 & 1132 & 971 & 16.5 \\
\hline
\end{tabular}

The shape and dimension of specimens for ultrasonic fatigue tests are shown in Fig. 1. These specimens have a diameter of $4 \mathrm{~mm}$ in the center. The surface of the specimens' central region was polished using abrasive paper with a grit scale of 500, 1000, 1500 and 2000 to remove machining marks that could lead to stress concentration and promote premature fracture. A set of specimens was subjected to SMAT with two levels of intensity. The SMAT process was performed at room temperature with $100 \mathrm{Cr} 6$ steel shot of $2 \mathrm{~mm}$ diameter at a sonotrode vibration amplitude of $20 \mu \mathrm{m}$ (denoted as SMATed 20) and $45 \mu \mathrm{m}$ (denoted as SMATed 45) for 20 minutes. A rotating motor with a speed of $80 \mathrm{rpm}$ was adapted to turn the specimens in order to get a homogenous treatment on the surface [13]. According to previous studies, a nanostructured layer can be generated on the top surface of the specimens, especially under high treatment intensity[11]. More detailed description and principle of SMAT can be found in $[13,15]$.

Fatigue experiments were conducted using an ultrasonic fatigue system at room temperature with a resonance frequency of $20 \mathrm{kHz}$, and a stress ratio of $\mathrm{R}=$ 1. During ultrasonic fatigue test, the specimens were cooled by compressed air, and ultrasonic interval loading was applied to avoid heating in order to keep specimen temperature below $35^{\circ} \mathrm{C}$.

After the fatigue tests, the fracture surfaces of failed specimens were observed using SEM, and the chemical composition of the crack initiation site on fracture surface was analyzed by Energy Dispersive X-ray Analysis (EDX) in the SEM.

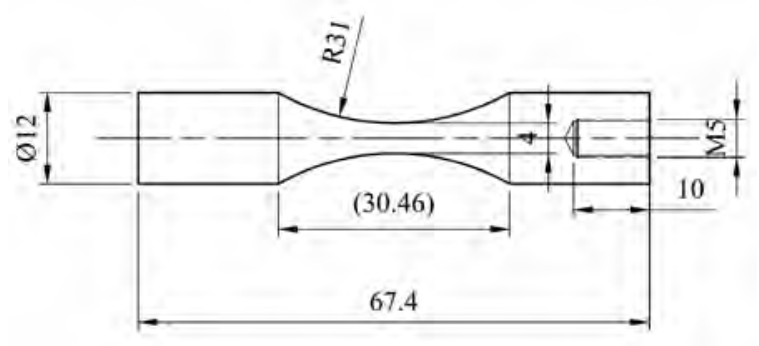

Fig. 1. Shape and dimension of specimens for ultrasonic fatigue tests

\section{Results and discussion}

\subsection{Nanostructured layer generated by SMAT}

There are many studies reported in the literature on surface nanocrystallisation of material surface by SMAT process. The deformation and nanocrystallisation of this alloy is essentially related to twinning and dislocation activities. The nanocrystallisation and grain refinement mechanism of titanium was investigated by Zhu et al. [16].

SEM micrographs of fracture surface of TC11 specimens processed with a sonotrode vibration amplitude of $20 \mu \mathrm{m}$ (SMATed 20) and $45 \mu \mathrm{m}$ (SMATed 45) are shown in Fig. 2. It is shown that a nanostructured and strongly plastically deformed layer generated by SMAT can be distinguished on the fracture surface, which is similar to $\mathrm{Ni}_{3} \mathrm{Al}$ [17] and $\mathrm{S} 45 \mathrm{C}$ steel [18] presented in the literature. When SMAT is carried out at a vibration amplitude of $20 \mu \mathrm{m}$, the nanostructured and strongly plastically deformed layer is generated in the near surface layer, and it seems there is a transition boundary between the strongly plastically deformed layer and the bulk, as shown in Fig. 2a. As the sonotrode vibration amplitude increased from $20 \mu \mathrm{m}$ to $45 \mu \mathrm{m}$, the thickness of the nanostructured and strongly plastically 
deformed layer is increased from approximately $6 \mu \mathrm{m}$ to $12 \mu \mathrm{m}$, which can be seen in Fig. 2b. Besides, there are no obvious boundary between deformable layer and the bulk for SMAT 45.
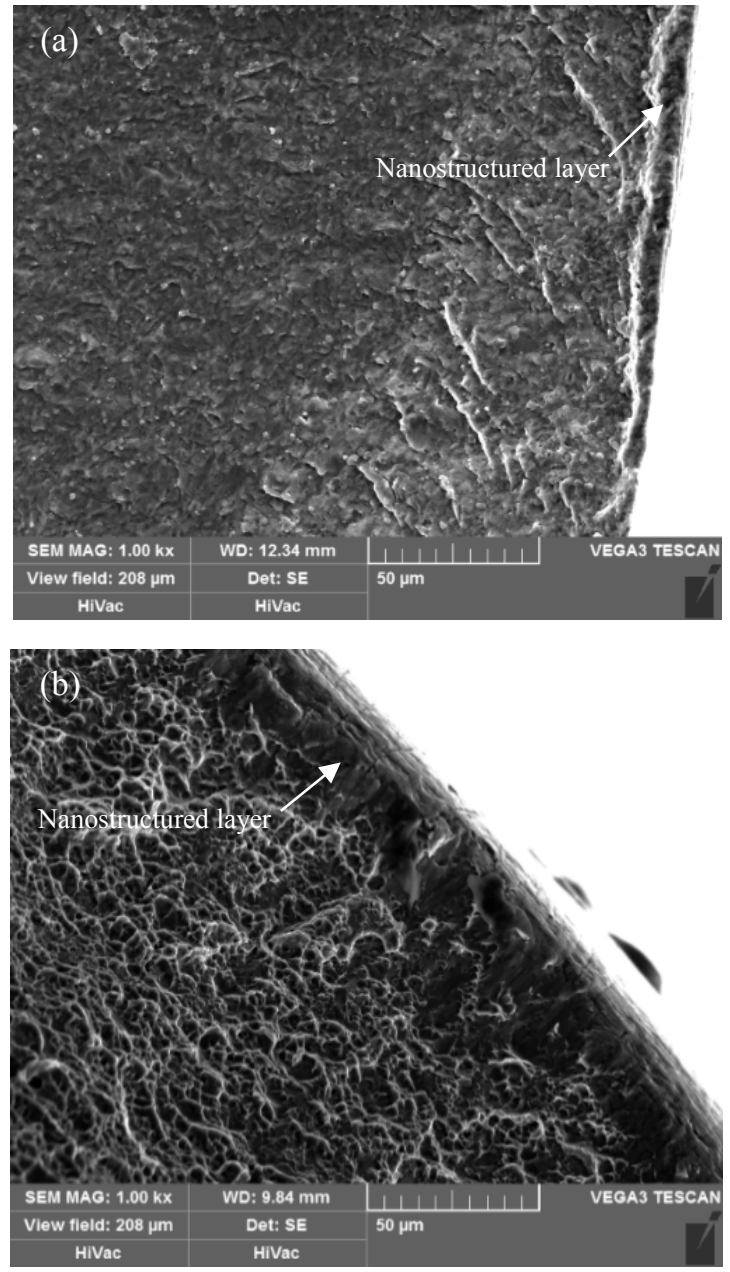

Fig. 2. SEM micrographs of fracture surface of SMATed TC11 specimens; nanostructured and strongly plastically deformed layer produced by SMAT with a sonotrode vibration amplitude of (a) $20 \mu \mathrm{m}$ and (b) $45 \mu \mathrm{m}$

\subsection{Fatigue life}

The ultrasonic fatigue test results of both untreated and SMATed samples are shown in Fig. 3 in the form of S-N plot. The surface and interior crack initiation of untreated specimens are represented by the hollow circles and solid circles, respectively. Triangles are used to indicate the fatigue test results of specimens SMATed with a sonotrode vibration amplitude of $20 \mu \mathrm{m}$ and 45 $\mu \mathrm{m}$, which are respectively referred to red and blue triangles. The fatigue life of the untreated specimens increases with the decrease of the applied stress, varying from $2 \times 10^{7}$ cycles to $1.7 \times 10^{9}$ cycles in a range of applied stress levels from 720 to $660 \mathrm{MPa}$. It seems that at a same stress level, specimens with surface crack initiation failed earlier than specimens with crack initiation sites located in the bulk. Unexpectedly the fatigue life and fatigue strength of the SMATed specimens significantly decrease compared to the untreated specimens, especially for higher vibration amplitude, (SMATed 45). Qualitatively this could be explained by a higher roughness of SMATed specimens due to shot impacts in comparison to the polished untreated specimens. As a matter of fact, higher SMAT intensity (represented by vibration amplitude) leads to higher surface roughness of the specimens. The higher roughness induced by SMAT could have played the role of a micro notch, and consequently promoted the crack nucleation at surface due to stress concentration. This assumption is consistent with the fact that the crack initiation occurred at surface for all the SMATed specimens, according to fracture surface observation using SEM. Deterioration in fatigue life by mechanical surface treatment was also reported by other authors [19]. In a further investigation, attention will be paid to the effect of SMAT on the crack initiation process of the TC11 alloy.

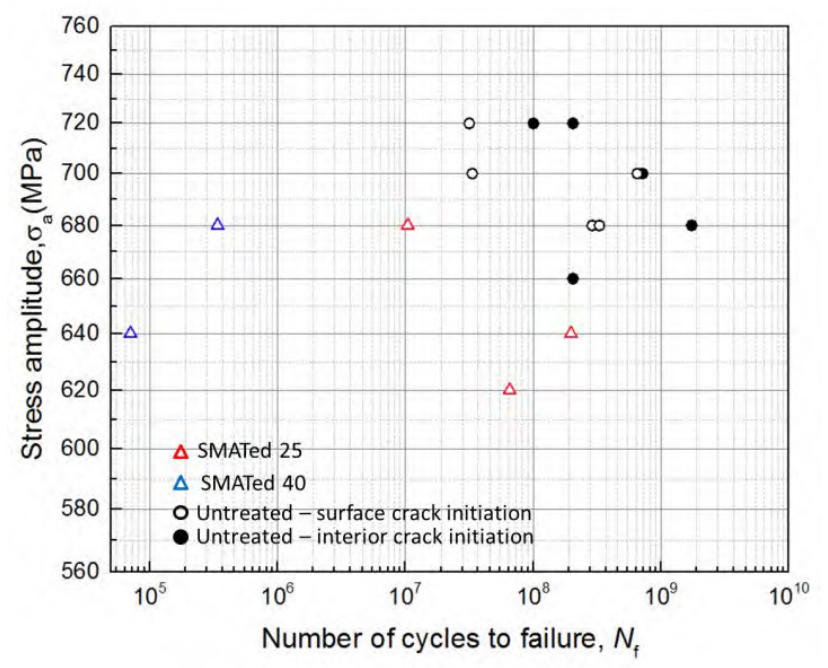

Fig. 3. S-N plot of the preliminary ultrasonic fatigue test results for the studied TC11 alloy.

\subsection{Microscopic morphology analysis of fracture surfaces}

The fatigue crack initiation site and the fracture surface morphology were observed by SEM. Based on the SEM observation of all the fracture surfaces, the crack initiation of untreated specimens can be classified into two crack initiation modes: surface induced failure and interior induced failure, as shown in Fig. 4a and Fig. 4b, respectively. The fatigue crack initiation of the titanium alloy studied in this work is similar to Ti-6Al-4V investigated by Zuo et al. [2]. The fracture surface morphologies revealed by SEM in the present work and in other investigations show that the location for main crack initiation in the VHCF regime is always determined by a competitive effect between the surface defects and the internal inhomogeneous microstructures such as grain orientation.

There is only one crack initiated and propagated through the cross section for surface induced failure of untreated specimens, as shown in Fig. 5a. The fracture surface shows radial lines and these lines radiate out from the center of the surface crack source position. Two 
types of surface crack initiation of Ti-6Al-4V alloy were observed in $[3,20]$, namely surface without facets (Type I) and surface with facets (Type II). Similar to the Ti$6 \mathrm{Al}-4 \mathrm{~V}$ alloy, both Type I and Type II can be found in the surface crack initiation area by using SEM observation. For type II, Fig. 5b shows that the diameter of the facets is about $6 \mu \mathrm{m}$. In the area close to the crack initiation center, there are a lot of small facets with approximately the same size. These facets should have been caused by the cleavage fracture of the $\alpha$ grains located in the subsurface region, according to the literature [3].
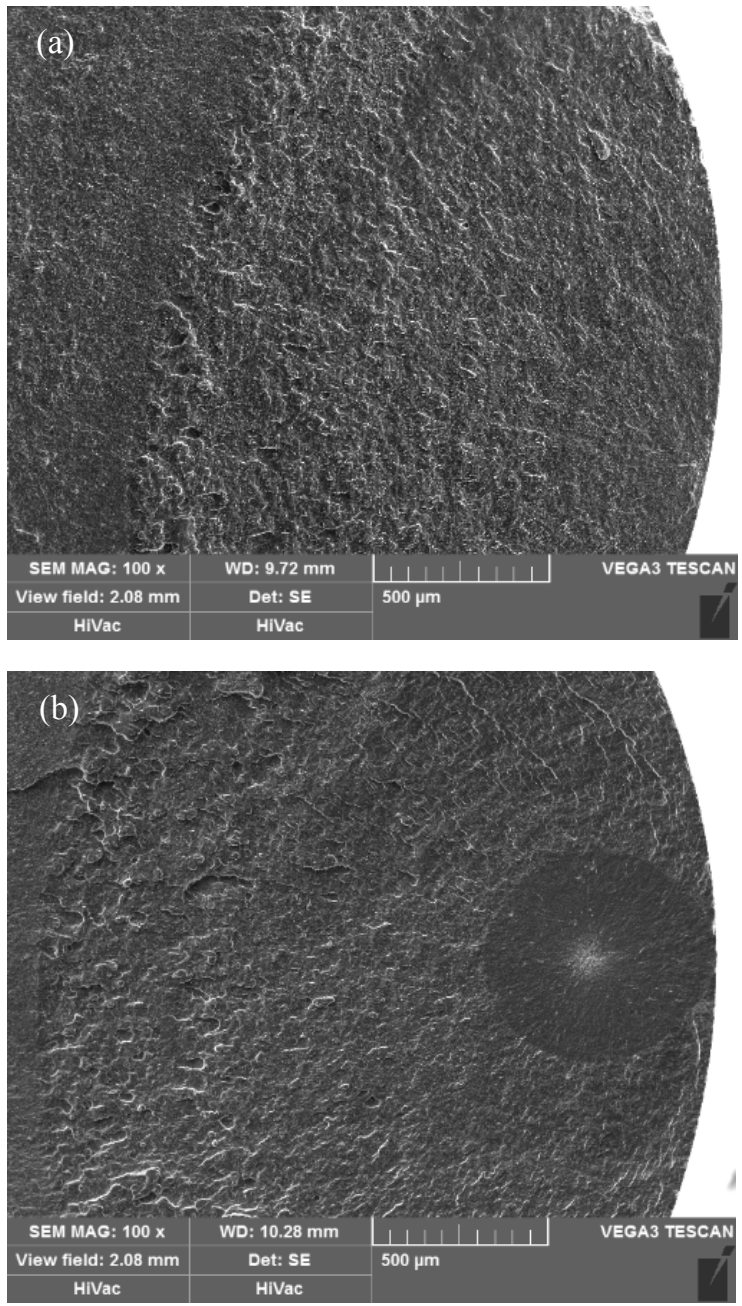

Fig. 4. SEM micrographs of (a) surface induced failure of a specimen without SMAT failed at $3.35 \times 10^{7}$ under $\sigma_{\mathrm{a}}=700 \mathrm{MPa}$, (b) interior induced failure of a specimen without SMAT failed at $1.27 \times 10^{8}$ under $\sigma_{\mathrm{a}}=660 \mathrm{MPa}$

Interior fatigue failure occurred at more than $10^{8}$ cycles and formed a typical fisheye area at the crack initiation site on the fracture surface. Fig. 6 shows an example of interior crack initiation of a specimen failed in the VHCF regime at $2.27 \times 10^{8}$ cycles under $660 \mathrm{MPa}$. A fine granular area (FGA) in the center of the fisheye region with similar fractographic characteristics as Ti6Al4V titanium alloy [1] is shown in Fig. 6a and Fig. 6b. The fisheye can be divided into two areas, namely FAG and flat surface around the FGA, which are marked in Fig. 6a. The FGA is approximated to a circle and the diameter is about $420 \mu \mathrm{m}$ with a very rough surface, which can be considered as the result of crack initiation process.

Fig. $6 \mathrm{~b}$ shows that there are no inclusions and pores in FGA. The chemical composition of the FGA was measured using electron dispersive spectroscopy (EDS). Fig. $6 \mathrm{c}$ shows that the chemical composition of this area is similar to that of the bulk material without inclusion. Therefore, the interior crack initiation of the present TC11 might be associated with microstructural inhomogeneities. This point will be checked in our future work.
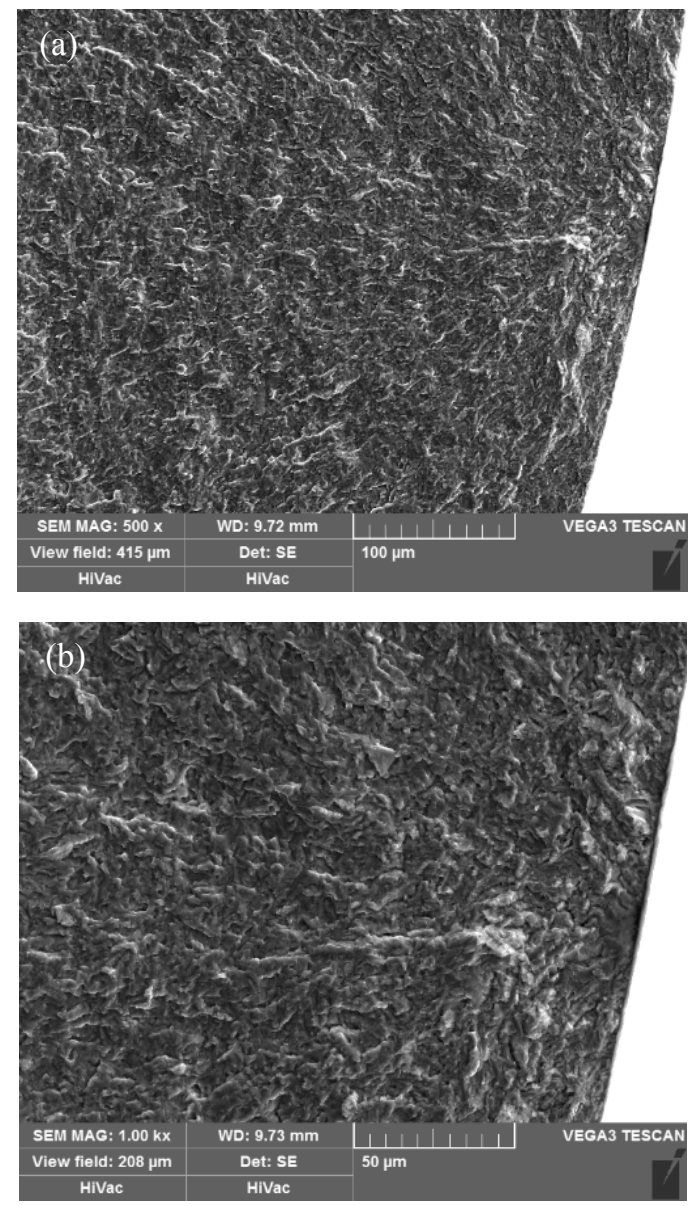

Fig. 5. (a) Crack initiation at the surface and (b) type II "surface with facets" of a specimen without SMAT failed at $2.90 \times 10^{8}$ under $\sigma_{\mathrm{a}}=680 \mathrm{MPa}$ 

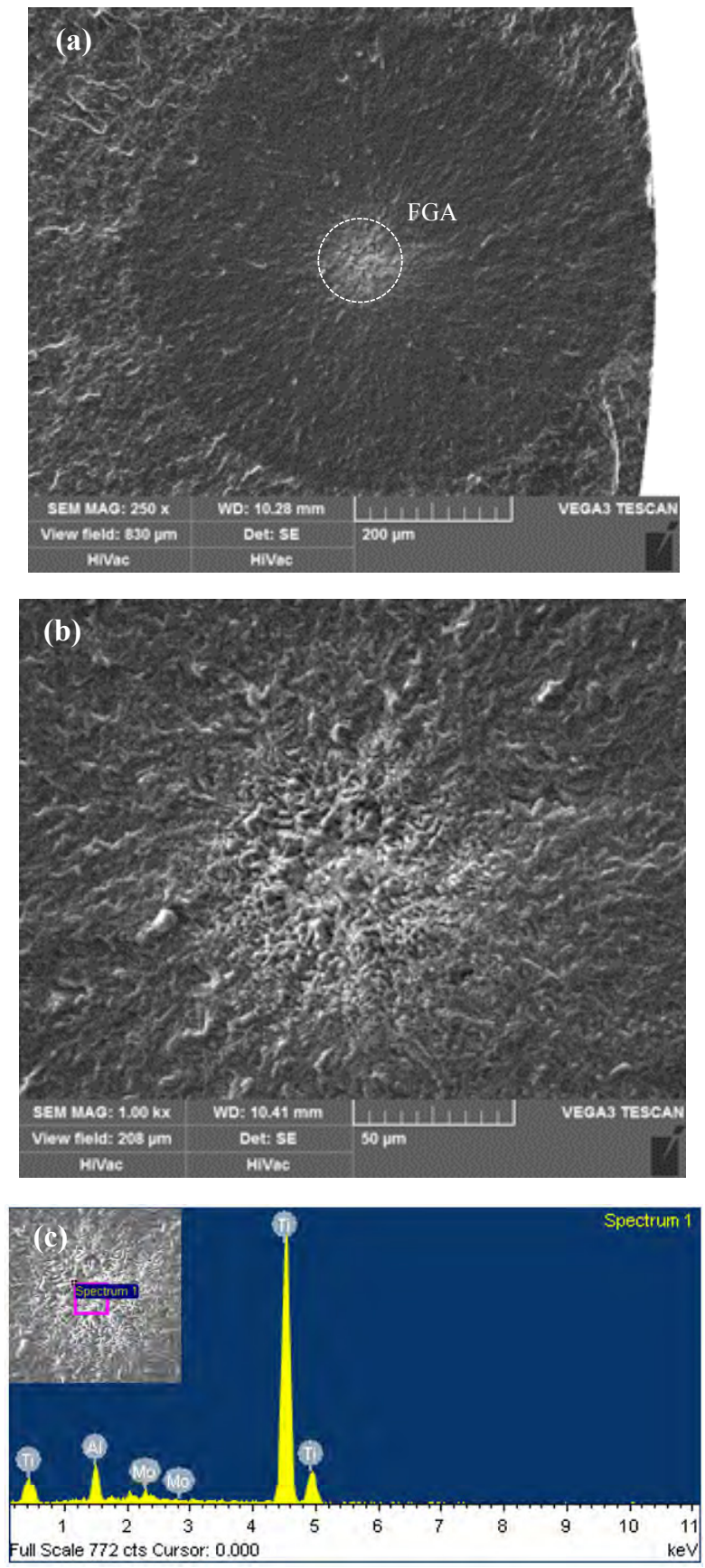

Fig. 6. (a) Fracture surface of interior induced failure with fisheye of a specimen failed at $2.05 \times 10^{8}$ under $\sigma_{\mathrm{a}}=720 \mathrm{MPa}$, (b) detailed observation of FGA (fine granular area), (c) chemical composition of the FGA analyzed using EDS

Furthermore, the surface roughness of the fisheye, as shown in Fig. 6, was analyzed by a surface profilometer (VeecoWyko NT1100) to derive quantitative geometric morphology information of the FGA and the flat surface in the fisheye region. Fig. 7 shows the 2D graph and the corresponding $3 \mathrm{D}$ scan from the surface profilometer of a $1.20 \mathrm{~mm} \times 0.93 \mathrm{~mm}$ area. The mean surface roughness $\mathrm{Ra}$ of the FGA and the other region in the fisheye is approximately $32 \mu \mathrm{m}$ and $3.4 \mu \mathrm{m}$, which was measured and calculated automatically by Vecco vision software.

In the case of specimens treated by SMAT, the fatigue failures are all initiated at the surface, as illustrated in Fig. 8a and Fig. 8b. The fatigue crack initiated at the surface of SMATed specimens without the presence of obvious facets. Compared to the surface initiation region of untreated specimens (Fig. 5), the surface crack initiation source of SMATed specimens is a larger area around the surface as shown in Fig. 8b. It is assumed that a micro notch caused by very rough surface induced by SMAT could be the main factor affecting the very high cycle fatigue life of the SMATed specimens.

Due to the fact that SMAT only affects surface and subsurface layers of specimens, the aspect of fatigue crack propagation should be similar to the case of the untreated specimens with surface-induced fatigue fracture. Fig. $8 \mathrm{c}$ is an example of fatigue crack propagation for failed specimens in the VHCF regime. In the crack propagation zone, many facets and second cracks can be observed with the presence of obvious typical characteristics of inter-crystalline and transcrystalline fatigue fracture in the VHCF regime.
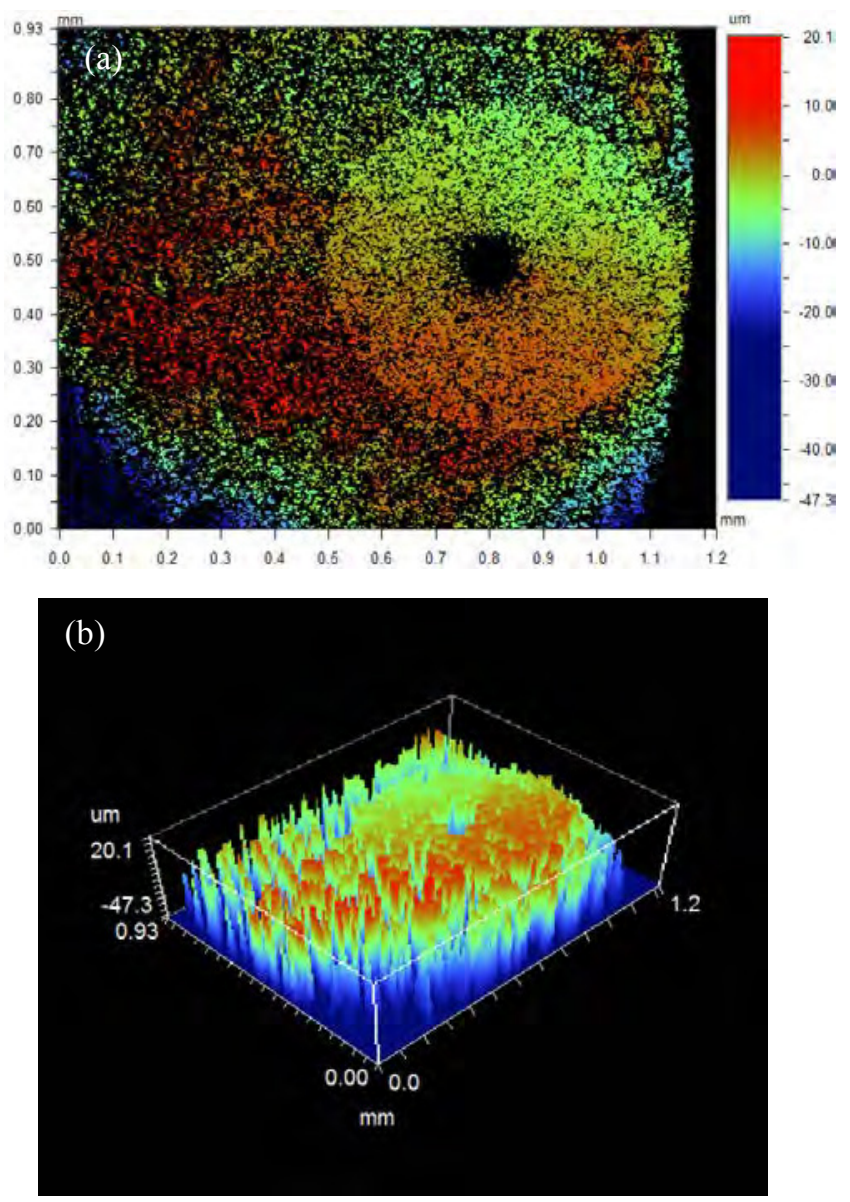

Fig. 7. (a) $2 \mathrm{D}$ and (b) $3 \mathrm{D}$ profiles of the fisheye of a specimen failed at $2.05 \times 10^{8}$ under $\sigma_{\mathrm{a}}=720 \mathrm{MPa}$ 

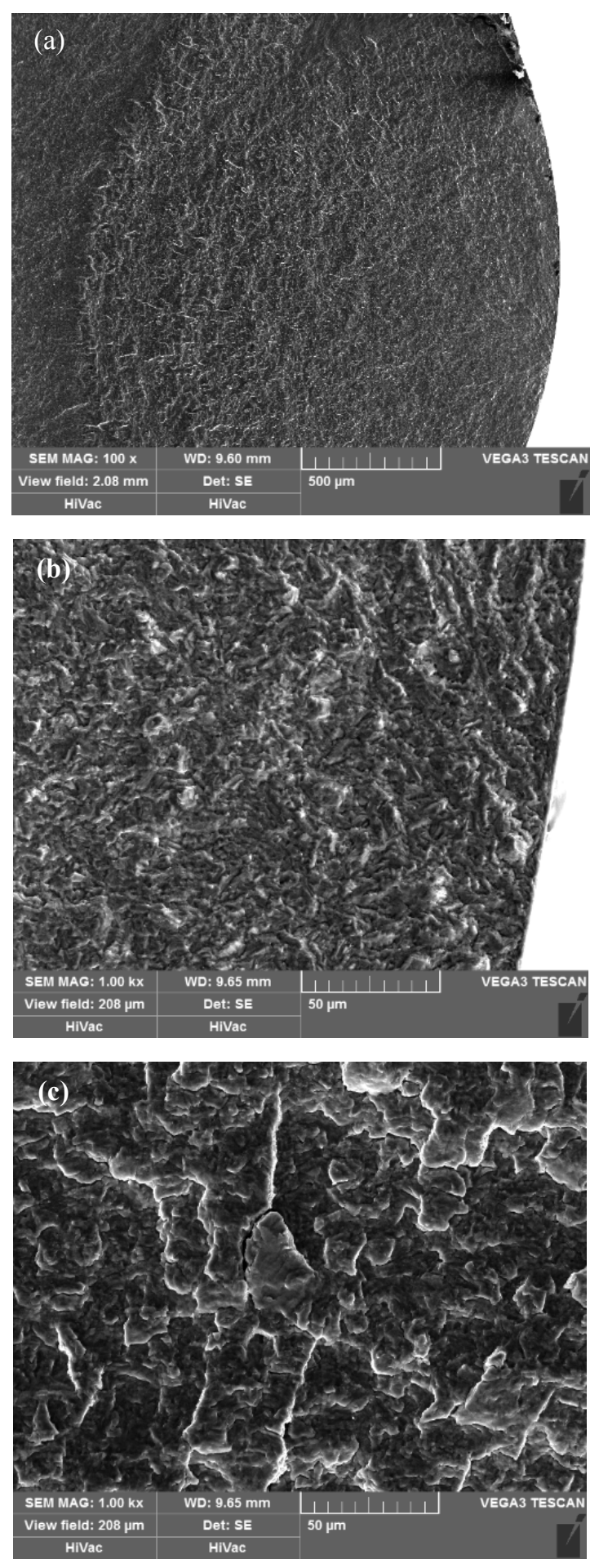

Fig. 8. (a) Fracture surface of a SMATed specimen (SMATed 45) failed at $1.05 \times 10^{7}$ under $\sigma_{\mathrm{a}}=680 \mathrm{MPa}$, (b) detail of the crack initiation source, (c) enlargement of the crack propagation zone

\section{Conclusion}

In this work, the fatigue behavior of a TC11 titanium alloy before and after SMAT has been studied in the very high cycle regime. A nanostructured and strongly plastically deformed layer has been generated in the surface region by SMAT. Contrary to the beneficial effect of SMAT in LCF and HCF regimes, the preliminary results presented in this paper show that SMAT has a negative effect on the fatigue properties under VHCF, It reduces the fatigue life compared to specimens without SMAT.

Two types of crack initiation modes, surface crack initiation and interior crack initiation in VHCF regime are observed for TC11 without SMAT. Interior fatigue failures occur at more than $10^{8}$ cycles and are characterized by the presence of fisheye. There are no inclusions and pores in FGA, and the interior crack initiation of TC11 in the VHCF regime could be related to microstructural inhomogeneities for example inhomogeneous grains orientation. The fatigue failures of SMATed specimens all initiate at the surface, and the fatigue crack propagation is the same as the surfaceinduced fatigue fracture of untreated specimens. The crack propagation zone shows obvious characteristics of inter-crystalline and trans-crystalline fatigue fracture.

\section{References}

1. S. Heinz, F. Balle, G. Wagner, D. Eifler, Ultrasonics 53(8) 1433-1440 (2013).

2. J. Zuo, Z. Wang, E. Han, Materials Science and Engineering: A 473(1) 147-152 (2008).

3. X. Liu, C. Sun, Y. Hong, Materials Science and Engineering: A 622 228-235 (2015).

4. Z.Y. Huang, H.Q. Liu, H.M. Wang, D. Wagner, M.K. Khan, Q.Y. Wang, International Journal of Fatigue 93 232-237 (2016).

5. Z. Sun, D. Retraint, T. Baudin, A. Helbert, F. Brisset, M. Chemkhi, J. Zhou, P. Kanouté, Materials Characterization 124 117-121 (2017).

6. J. Zhou, Z. Sun, P. Kanouté, D. Retraint, International Journal of Fatigue $\mathbf{1 0 3} \quad$ 309-317 (2017).

7. K. Lu, J. Lu, Materials Science and Engineering: A 375 38-45 (2004).

8. Y. Liu, B. Jin, J. Lu, Materials Science and Engineering: A 636 446-451 (2015).

9. T. Roland, D. Retraint, K. Lu, J. Lu, Materials Science Forum 490-491 625-630 (2005).

10. G. Proust, P. Trimby, S. Piazolo, D. Retraint, Journal of Visualized Experiments : JoVE (122) 55506 (2017).

11. Y. Pi, G. Agoda-Tandjawa, S. Potiron, C. Demangel, D. Retraint, H. Benhayoune, Journal of Nanoscience and Nanotechnology 12(6) 4892-4897 (2012).

12. L. Yang, N.R. Tao, K. Lu, L. Lu, Scripta Materialia 68(10) 801-804 (2013).

13. T. Roland, D. Retraint, K. Lu, J. Lu, Scripta Materialia 54(11) 1949-1954 (2006).

14. S.A. Kumar, S.G.S. Raman, T.S. Narayanan, Transactions of the Indian Institute of Metals 67(1) 137-141 (2014). 
15. Sandá, V.G. Navas, O. Gonzalo, Materials \& Design 32(4) 2213-2220 (2011).

16. K. Zhu, A. Vassel, F. Brisset, K. Lu, J. Lu, Acta Materialia 52(14) 4101-4110 (2004).

17. J. Ren, A. Shan, J. Zhang, H. Song, J. Liu, Materials Letters 60(17) 2076-2079 (2006).

18. X. Cao, Y. Pyoun, R. Murakami, Applied Surface Science 256(21) 6297-6303 (2010).

19. M.-S. Suh, C.H. Suh, S.-H. Nahm, C.-M. Suh, Advances in Mechanical Engineering 7(1) 126848 (2015).

20. V. Crupi, G. Epasto, E. Guglielmino, A. Squillace, International Journal of Fatigue 95 64-75 (2017). 